\title{
The ternary Goldbach problem with prime numbers of a mixed type
}

\author{
S. I. Dimitrov \\ Faculty of Applied Mathematics and Informatics, Technical University of Sofia \\ 8 St. Kliment Ohridski Blvd., 1756 Sofia, Bulgaria \\ e-mail: sdimitrovetu-sofia.bg
}

Abstract: In the present paper we prove that every sufficiently large odd integer $N$ can be represented in the form

$$
N=p_{1}+p_{2}+p_{3},
$$

where $p_{1}, p_{2}, p_{3}$ are primes, such that $p_{1}=x^{2}+y^{2}+1, p_{2}=\left[n^{c}\right]$.

Keywords: Goldbach problem, Prime numbers, Circle method.

2010 Mathematics Subject Classification: 11N36, 11P32.

\section{Notations}

Let $N$ be a sufficiently large odd integer. The letter $p$, with or without subscript, will always denote prime numbers. Let $A>100$ be a constant. By $\varepsilon$ we denote an arbitrary small positive number, not the same in all appearances. The relation $f(x) \ll g(x)$ means that $f(x)=\mathcal{O}(g(x))$. As usual $[t]$ and $\{t\}$ denote the integer part, respectively, the fractional part of $t$. Instead of $m \equiv n(\bmod k)$ we write for simplicity $m \equiv n(k)$. As usual $e(t)=\exp (2 \pi i t)$. We denote by $(d, q),[d, q]$ the greatest common divisor and the least common multiple of $d$ and $q$ respectively. As usual $\varphi(d)$ is Euler's function; $\mu(d)$ is Möbius' function; $r(d)$ is the number of solutions of the equation $d=m_{1}^{2}+m_{2}^{2}$ in integers $m_{j} ; \chi(d)$ is the non-principal character modulo 4 and $L(s, \chi)$ is the corresponding Dirichlet's $L$-function. By $c_{0}$ we denote some positive number, not necessarily the same in different occurrences. Let $c$ be a real constant such that $1<c<73 / 64$. 


\section{Denote}

$$
\begin{aligned}
& \gamma=1 / c \\
& D=\frac{N^{1 / 2}}{(\log N)^{A}} ; \\
& \psi(t)=\{t\}-1 / 2 \\
& \theta_{0}=\frac{1}{2}-\frac{1}{4} e \log 2=0.0289 \ldots \text {; } \\
& \mathfrak{S}_{d, l}(N)=\prod_{\substack{p \nmid d \\
p \mid N}}\left(1-\frac{1}{(p-1)^{2}}\right) \prod_{\substack{p \mid d \\
p \nmid N-l}}\left(1-\frac{1}{(p-1)^{2}}\right) \\
& \times \prod_{p \nmid d N}\left(1+\frac{1}{(p-1)^{3}}\right) \prod_{\substack{p|d \\
p| N-l}}\left(1+\frac{1}{p-1}\right) ; \\
& \mathfrak{S}(N)=\prod_{p \mid N}\left(1-\frac{1}{(p-1)^{2}}\right) \prod_{p \nmid N}\left(1+\frac{1}{(p-1)^{3}}\right) ; \\
& \mathfrak{S}_{\Gamma}(N)=\pi \mathfrak{S}(N) \prod_{p \nmid N(N-1)}\left(1+\chi(p) \frac{p-3}{p\left(p^{2}-3 p+3\right)}\right) \prod_{p \mid N}\left(1+\chi(p) \frac{1}{p(p-1)}\right) \\
& \times \prod_{p \mid N-1}\left(1+\chi(p) \frac{2 p-3}{p\left(p^{2}-3 p+3\right)}\right) \text {; } \\
& \Delta(t, h)=\max _{y \leq t} \max _{(l, h)=1}\left|\sum_{\substack{p \leq y \\
p \equiv l(h)}} \log p-\frac{y}{\varphi(h)}\right|
\end{aligned}
$$

\section{Introduction and statement of the result}

In 1937, I. M. Vinogradov [15] solved the ternary Goldbach problem. He proved that for a sufficiently large odd integer $N$

$$
\sum_{p_{1}+p_{2}+p_{3}=N} \log p_{1} \log p_{2} \log p_{3}=\frac{1}{2} \mathfrak{S}(N) N^{2}+\mathcal{O}\left(\frac{N^{2}}{\log ^{A} N}\right),
$$

where $\mathfrak{S}(N)$ is defined by (6) and $A>0$ is an arbitrarily large constant.

In 1953, Piatetski-Shapiro [9] proved that for any fixed $c \in(1,12 / 11)$ the sequence

$$
\left(\left[n^{c}\right]\right)_{n \in \mathbb{N}}
$$

contains infinitely many prime numbers. Such prime numbers are named in honor of PiatetskiShapiro. The interval for $c$ was subsequently improved many times and the best result up to now belongs to Rivat and $\mathrm{Wu}[10]$ for $c \in(1,243 / 205)$. 
In 1992, A. Balog and J. P. Friedlander [1] considered the ternary Goldbach problem with variables restricted to Piatetski-Shapiro primes. They proved that, for any fixed $1<c<21 / 20$, every sufficiently large odd integer $N$ can be represented in the form

$$
N=p_{1}+p_{2}+p_{3}
$$

where $p_{1}, p_{2}, p_{3}$ are primes, such that $p_{k}=\left[n_{k}^{c}\right], \mathrm{k}=1,2,3$. Rivat [10] extended the range to $1<c<199 / 188$; Kumchev [7] extended the range to $1<c<53 / 50$. Jia [5] used a sieve method to enlarge the range to $1<c<16 / 15$.

Furthermore, Kumchev [7] proved that for any fixed $1<c<73 / 64$ every sufficiently large odd integer may be written as the sum of two primes and prime number of type $p=\left[n^{c}\right]$.

On the other hand, in 1960, Linnik [8] showed that there exist infinitely many prime numbers of the form $p=x^{2}+y^{2}+1$, where $x$ and $y$ are integers. In 2010 Tolev [14] proved that every sufficiently large odd integer $N$ can be represented in the form

$$
N=p_{1}+p_{2}+p_{3}
$$

where $p_{1}, p_{2}, p_{3}$ are primes, such that $p_{k}=x_{k}^{2}+y_{k}^{2}+1, \mathrm{k}=1,2$. In 2017 Teräväinen [12] improved Tolev's result for primes $p_{1}, p_{2}, p_{3}$, such that $p_{k}=x_{k}^{2}+y_{k}^{2}+1, k=1,2,3$.

Recently the author [2] proved that there exist infinitely many arithmetic progressions of three different primes $p_{1}, p_{2}, p_{3}=2 p_{2}-p_{1}$ such that $p_{1}=x^{2}+y^{2}+1, p_{3}=\left[n^{c}\right]$.

Define

$$
\Gamma(N)=\sum_{\substack{p_{1}+p_{2}+p_{3}=N \\ p_{2}=\left[n^{c}\right]}} r\left(p_{1}-1\right) p_{2}^{1-\gamma} \log p_{1} \log p_{2} \log p_{3} .
$$

Motivated by these results we shall prove the following theorem.

Theorem 1. Assume that $1<c<73 / 64$. Then the asymptotic formula

$$
\Gamma(N)=\frac{\gamma}{2} \mathfrak{S}_{\Gamma}(N) N^{2}+\mathcal{O}\left(N^{2}(\log N)^{-\theta_{0}}(\log \log N)^{6}\right)
$$

holds. Here $\gamma, \theta_{0}$ and $\mathfrak{S}_{\Gamma}(N)$ are defined by (1), (4) and (7).

Bearing in mind that $\mathfrak{S}_{\Gamma}(N) \gg 1$ for $N$ odd, from Theorem 1 it follows that for any fixed $1<c<73 / 64$ every sufficiently large odd integer $N$ can be written in the form

$$
N=p_{1}+p_{2}+p_{3}
$$

where $p_{1}, p_{2}, p_{3}$ are primes, such that $p_{1}=x^{2}+y^{2}+1, p_{2}=\left[n^{c}\right]$.

The asymptotic formula obtained for $\Gamma(N)$ is the product of the individual asymptotic formulas

$$
\sum_{p_{1}+p_{2}+p_{3}=N} r\left(p_{1}-1\right) \log p_{1} \log p_{2} \log p_{3} \sim \frac{1}{2} \mathfrak{S}_{\Gamma}(N) N^{2}
$$

and

$$
\frac{1}{N} \sum_{\substack{p \leq N \\ p=\left[n^{c}\right]}} p^{1-\gamma} \log p \sim \gamma
$$

The proof of Theorem 1 follows the same ideas as the proof in [2]. 


\section{Outline of the proof}

Using (10) and well-known identity $r(n)=4 \sum_{d \mid n} \chi(d)$ we find

$$
\Gamma(N)=4\left(\Gamma_{1}(N)+\Gamma_{2}(N)+\Gamma_{3}(N)\right),
$$

where

$$
\begin{aligned}
& \Gamma_{1}(N)=\sum_{\substack{p_{1}+p_{2}+p_{3}=N \\
p_{2}=\left[n^{c}\right]}}\left(\sum_{\substack{d \mid p_{1}-1 \\
d \leq D}} \chi(d)\right) p_{2}^{1-\gamma} \log p_{1} \log p_{2} \log p_{3}, \\
& \Gamma_{2}(N)=\sum_{\substack{p_{1}+p_{2}+p_{3}=N \\
p_{2}=[n c]}}\left(\sum_{\substack{d \mid p_{1}-1 \\
D<d<N / D}} \chi(d)\right) p_{2}^{1-\gamma} \log p_{1} \log p_{2} \log p_{3}, \\
& \Gamma_{3}(N)=\sum_{\substack{p_{1}+p_{2}+p_{3}=N \\
p_{2}=\left[n^{c}\right]}}\left(\sum_{\substack{d \mid p_{1}-1 \\
d \geq N / D}} \chi(d)\right) p_{2}^{1-\gamma} \log p_{1} \log p_{2} \log p_{3} .
\end{aligned}
$$

In order to estimate $\Gamma_{1}(N)$ and $\Gamma_{3}(N)$ we have to consider the sum

$$
I_{d, l ; J}(N)=\sum_{\substack{p_{1}+p_{2}+p_{3}=N \\ p_{1}=l(d) \\ p_{1} \in J \\ p_{2}=\left[n^{c}\right]}} p_{2}^{1-\gamma} \log p_{1} \log p_{2} \log p_{3},
$$

where $d$ and $l$ are coprime natural numbers, and $J \subset[1, N]$. The left and the right side of the interval $J$, we shall denote with $J_{1}$ and $J_{2}$, i.e. $J=\left(J_{1}, J_{2}\right]$. If $J=[1, N]$ then we write for simplicity $I_{d, l}(N)$. We apply the circle method. Clearly

$$
I_{d, l ; J}(N)=\int_{0}^{1} S_{d, l ; J}(\alpha) S(\alpha) S_{c}(\alpha) e(-N \alpha) d \alpha,
$$

where

$$
\begin{aligned}
& S_{d, l ; J}(\alpha)=\sum_{\substack{p \in J \\
p \equiv l(d)}} e(\alpha p) \log p, \\
& S(\alpha)=S_{1,1 ;[1, N]}(\alpha), \\
& S_{c}(\alpha)=\sum_{\substack{p \leq N \\
p=\left[n^{c}\right]}} p^{1-\gamma} e(\alpha p) \log p .
\end{aligned}
$$

We define major and minor arcs by

$$
E_{1}=\bigcup_{q \leq Q} \bigcup_{\substack{a=0 \\(a, q)=1}}^{q-1}\left[\frac{a}{q}-\frac{1}{q \tau}, \frac{a}{q}+\frac{1}{q \tau}\right], E_{2}=\left[\frac{1}{\tau}, 1+\frac{1}{\tau}\right] \backslash E_{1},
$$


where

$$
Q=(\log N)^{B}, \quad \tau=N Q^{-1}, A>4 B+3, B>14 .
$$

Then we have the decomposition

$$
I_{d, l ; J}(N)=I_{d, l ; J}^{(1)}(N)+I_{d, l ; J}^{(2)}(N)
$$

where

$$
I_{d, l ; J}^{(i)}(N)=\int_{E_{i}} S_{d, l ; J}(\alpha) S(\alpha) S_{c}(\alpha) e(-N \alpha) d \alpha, \quad i=1,2 .
$$

We shall estimate $I_{d, l ; J}^{(1)}(N), \Gamma_{3}(N), \Gamma_{2}(N)$ and $\Gamma_{1}(N)$ respectively in the sections $4,5,6$ and 7. In section 8 we shall complete the proof of the Theorem.

\section{Asymptotic formula for $I_{d, l ; J}^{(1)}(N)$}

We have

$$
I_{d, l ; J}^{(1)}(N)=\sum_{q \leq Q} \sum_{\substack{a=0 \\(a, q)=1}}^{q-1} H(a, q)
$$

where

$$
H(a, q)=\int_{-1 / q \tau}^{1 / q \tau} S_{d, l ; J}\left(\frac{a}{q}+\alpha\right) S\left(\frac{a}{q}+\alpha\right) S_{c}\left(\frac{a}{q}+\alpha\right) e\left(-N\left(\frac{a}{q}+\alpha\right)\right) d \alpha .
$$

On the other hand,

$$
S_{d, l ; J}\left(\frac{a}{q}+\alpha\right)=\sum_{\substack{1 \leq m \leq q \\(m, q)=1 \\ m \equiv l((d, q))}} e\left(\frac{a m}{q}\right) T(\alpha)+\mathcal{O}(q \log N)
$$

where

$$
T(\alpha)=\sum_{\substack{p \in J \\ p=l(d) \\ p \equiv m(q)}} e(\alpha p) \log p .
$$

According to the Chinese remainder theorem there exists integer $f=f(l, m, d, q)$ such that $(f,[d, q])=1$ and

$$
T(\alpha)=\sum_{\substack{p \in J \\ p \equiv f([d, q])}} e(\alpha p) \log p
$$


Applying Abel's transformation we obtain

$$
\begin{aligned}
T(\alpha) & =-\int_{J_{1}}^{J_{2}}\left(\sum_{\substack{J_{1}<p<t \\
p \equiv f([d, q])}} \log p\right) \frac{d}{d t}(e(\alpha t)) d t+\left(\sum_{\substack{p \in J \\
p \equiv f([d, q])}} \log p\right) e\left(\alpha J_{2}\right) \\
& =-\int_{J_{1}}^{J_{2}}\left(\frac{t-J_{1}}{\varphi([d, q])}+\mathcal{O}\left(\Delta\left(J_{2},[d, q]\right)\right)\right) \frac{d}{d t}(e(\alpha t)) d t \\
& +\left(\frac{J_{2}-J_{1}}{\varphi([d, q])}+\mathcal{O}\left(\Delta\left(J_{2},[d, q]\right)\right)\right) e\left(\alpha J_{2}\right) \\
& =\frac{1}{\varphi([d, q])} \int_{J_{1}}^{J_{2}} e(\alpha t) d t+\mathcal{O}\left(\left(1+|\alpha|\left(J_{2}-J_{1}\right)\right) \Delta\left(J_{2},[d, q]\right)\right) .
\end{aligned}
$$

We use the well known formula

$$
\int_{J_{1}}^{J_{2}} e(\alpha t) d t=M_{J}(\alpha)+\mathcal{O}(1)
$$

where

$$
M_{J}(\alpha)=\sum_{m \in J} e(\alpha m) .
$$

Bearing in mind that $|\alpha| \leq 1 / q \tau$ and $J \subset(1, N]$, from (21), (27) and (28) we get

$$
T(\alpha)=\frac{M_{J}(\alpha)}{\varphi([d, q])}+\mathcal{O}\left(\left(1+\frac{Q}{q}\right) \Delta(N,[d, q])\right) .
$$

From (26) and (29) it follows

$$
S_{d, l ; J}\left(\frac{a}{q}+\alpha\right)=\frac{c_{d}(a, q, l)}{\varphi([d, q])} M_{J}(\alpha)+\mathcal{O}(Q(\log N) \Delta(N,[d, q])),
$$

where

$$
c_{d}(a, q, l)=\sum_{\substack{1 \leq m \leq q \\(m, q)=1 \\ m \equiv l((d, q))}} e\left(\frac{a m}{q}\right)
$$

We shall find asymptotic formula for $S_{c}\left(\frac{a}{q}+\alpha\right)$. From (19) we have

$$
\begin{aligned}
S_{c}(\alpha) & =\sum_{p \leq N} p^{1-\gamma}\left(\left[-p^{\gamma}\right]-\left[-(p+1)^{\gamma}\right]\right) e(\alpha p) \log p \\
& =\Omega(\alpha)+\Sigma(\alpha),
\end{aligned}
$$

where

$$
\begin{aligned}
& \Omega(\alpha)=\sum_{p \leq N} p^{1-\gamma}\left((p+1)^{\gamma}-p^{\gamma}\right) e(\alpha p) \log p \\
& \Sigma(\alpha)=\sum_{p \leq N} p^{1-\gamma}\left(\psi\left(-(p+1)^{\gamma}\right)-\psi\left(-p^{\gamma}\right)\right) e(\alpha p) \log p .
\end{aligned}
$$


According to Kumchev ([7], Theorem 2) for $64 / 73<\gamma<1$ uniformly in $\alpha$ we have that

$$
\Sigma\left(\frac{a}{q}+\alpha\right) \ll N^{1-\varepsilon}
$$

On the other hand,

$$
(p+1)^{\gamma}-p^{\gamma}=\gamma p^{\gamma-1}+\mathcal{O}\left(p^{\gamma-2}\right) .
$$

The formulas (32) and (35) give us

$$
\Omega(\alpha)=\gamma S(\alpha)+\mathcal{O}\left(N^{\varepsilon}\right)
$$

where $S(\alpha)$ is defined by (18).

According to ([6], Lemma 3, §10) we have

$$
S\left(\frac{a}{q}+\alpha\right)=\frac{\mu(q)}{\varphi(q)} M(\alpha)+\mathcal{O}\left(N e^{-c_{0} \sqrt{\log N}}\right)
$$

where

$$
M(\alpha)=\sum_{m \leq N} e(\alpha m) .
$$

Bearing in mind (31), (34), (36) and (37) we obtain

$$
S_{c}\left(\frac{a}{q}+\alpha\right)=\gamma \frac{\mu(q)}{\varphi(q)} M(\alpha)+\mathcal{O}\left(N e^{-c_{0} \sqrt{\log N}}\right)
$$

Furthermore, we need the trivial estimates

$\left|S_{d, l ; J}\left(\frac{a}{q}+\alpha\right)\right| \ll \frac{N \log N}{d}, \quad\left|S\left(\frac{a}{q}+\alpha\right)\right| \ll N, \quad|M(\alpha)| \ll N, \quad|\mu(q)| \ll 1$.

By (30), (37) - (39) and the well-known inequality $\varphi(n) \gg n(\log \log n)^{-1}$ we find

$$
\begin{aligned}
& S_{d, l ; J}\left(\frac{a}{q}+\alpha\right) S\left(\frac{a}{q}+\alpha\right) S_{c}\left(\frac{a}{q}+\alpha\right) e\left(-N\left(\frac{a}{q}+\alpha\right)\right) \\
& =\gamma \frac{c_{d}(a, q, l) \mu^{2}(q)}{\varphi([d, q]) \varphi^{2}(q)} M_{J}(\alpha) M^{2}(\alpha) e\left(-N\left(\frac{a}{q}+\alpha\right)\right)+\mathcal{O}\left(\frac{N^{3}}{d} e^{-c_{0} \sqrt{\log N}}\right) \\
& +\mathcal{O}\left(\frac{N^{2} Q \log ^{2} N}{q^{2}} \Delta(N,[d, q])\right) .
\end{aligned}
$$

Having in mind (21), (25) and (40) we get

$$
\begin{aligned}
H(a, q) & =\gamma \frac{c_{d}(a, q, l) \mu^{2}(q)}{\varphi([d, q]) \varphi^{2}(q)} e\left(-N \frac{a}{q}\right) \int_{-1 / q \tau}^{1 / q \tau} M_{J}(\alpha) M^{2}(\alpha) e(-N \alpha) d \alpha \\
& +\mathcal{O}\left(\frac{N^{2}}{q d} e^{-c_{0} \sqrt{\log N}}\right)+\mathcal{O}\left(\frac{N Q^{2} \log ^{2} N}{q^{3}} \Delta(N,[d, q])\right) .
\end{aligned}
$$


Taking into account (24), (41) and following the method in [13] we obtain

$$
\begin{aligned}
I_{d, l ; J}^{(1)}(N) & =\gamma \frac{\mathfrak{S}_{d, l}(N)}{\varphi(d)} \sum_{\substack{m_{1}+m_{2}+m_{3}=N \\
m_{1} \in J}} 1+\mathcal{O}\left(\frac{N^{2}}{d}(\log N) \sum_{q>Q} \frac{(d, q) \log q}{q^{2}}\right) \\
& +\mathcal{O}\left(\tau^{2}(\log N) \sum_{q \leq Q} \frac{q}{[d, q]}\right)+\mathcal{O}\left(N Q^{2}(\log N)^{2} \sum_{q \leq Q} \frac{\Delta(N,[d, q])}{q^{2}}\right) \\
& +\mathcal{O}\left(\frac{N^{2}}{d} e^{-c_{0} \sqrt{\log N}}\right),
\end{aligned}
$$

where $\mathfrak{S}_{d, l}(N)$ is defined by (5).

\section{Upper bound for $\Gamma_{3}(\mathrm{~N})$}

Consider the sum $\Gamma_{3}(N)$.

Since

$$
\sum_{\substack{d \mid p_{1}-1 \\ d \geq N / D}} \chi(d)=\sum_{\substack{m \mid p_{1}-1 \\ m \leq\left(p_{1}-1\right) D / N}} \chi\left(\frac{p_{1}-1}{m}\right)=\sum_{j= \pm 1} \chi(j) \sum_{\substack{m \mid p_{1}-1 \\ m \leq\left(p_{1}-1\right) D / N \\ \frac{p_{1}-1}{m} \equiv j(4)}} 1
$$

then from (14) and (15) it follows

$$
\Gamma_{3}(N)=\sum_{\substack{m<D \\ 2 \mid m}} \sum_{j= \pm 1} \chi(j) I_{4 m, 1+j m ; J_{m}}(N),
$$

where $J_{m}=[1+m N / D, N]$.

Therefore from (22) we get

$$
\Gamma_{3}(N)=\Gamma_{3}^{(1)}(N)+\Gamma_{3}^{(2)}(N),
$$

where

$$
\Gamma_{3}^{(\nu)}(N)=\sum_{\substack{m<D \\ 2 \mid m}} \sum_{j= \pm 1} \chi(j) I_{4 m, 1+j m ; J_{m}}^{(\nu)}(N), \quad \nu=1,2 .
$$

Let us consider first $\Gamma_{3}^{(2)}(N)$. Bearing in mind (23) for $i=2$ and (44) for $\nu=2$ we have

$$
\Gamma_{3}^{(2)}(N)=\int_{E_{2}} K(\alpha) S(\alpha) S_{c}(\alpha) e(-N \alpha) d \alpha,
$$

where

$$
K(\alpha)=\sum_{\substack{m<D \\ 2 \mid m}} \sum_{j= \pm 1} \chi(j) S_{4 m, 1+j m ; J_{m}}(\alpha)
$$

Using Cauchy's inequality we obtain

$$
\begin{aligned}
\Gamma_{3}^{(2)}(N) & \ll \sup _{\alpha \in E_{2} \backslash\{1\}}\left|S_{c}(\alpha)\right| \int_{E_{2}}|K(\alpha) S(\alpha)| d \alpha+\mathcal{O}\left(N^{\varepsilon}\right) \\
& \ll \sup _{\alpha \in E_{2} \backslash\{1\}}\left|S_{c}(\alpha)\right|\left(\int_{0}^{1}|K(\alpha)|^{2} d \alpha\right)^{1 / 2}\left(\int_{0}^{1}|S(\alpha)|^{2} d \alpha\right)^{1 / 2}+\mathcal{O}\left(N^{\varepsilon}\right) .
\end{aligned}
$$


From (31) and (36) we have

$$
S_{c}(\alpha)=\gamma S(\alpha)+\Sigma(\alpha)+\mathcal{O}\left(N^{\varepsilon}\right),
$$

where $S(\alpha)$ and $\Sigma(\alpha)$ are defined by (18) and (33).

Using (20) and (21) we can prove in the same way as in ([6], Ch.10, Th.3) that

$$
\sup _{\alpha \in E_{2} \backslash\{1\}}|S(\alpha)| \ll \frac{N}{(\log N)^{B / 2-4}} .
$$

According to Kumchev ([7], Theorem 2) we have that

$$
\sup _{\alpha \in E_{2} \backslash\{1\}}|\Sigma(\alpha)| \ll N^{1-\varepsilon} .
$$

Bearing in mind (47)-(49) we get

$$
\sup _{\alpha \in E_{2} \backslash\{1\}}\left|S_{c}(\alpha)\right| \ll \frac{N}{(\log N)^{B / 2-4}} .
$$

From (18) after straightforward computations we find

$$
\int_{0}^{1}|S(\alpha)|^{2} d \alpha \ll N \log N .
$$

On the other hand, from (17) and (45) we obtain

$$
\begin{aligned}
& \int_{0}^{1}|K(\alpha)|^{2} d \alpha=\sum_{\substack{m_{1}, m_{2}<D \\
2\left|m_{1}, 2\right| m_{2}}} \sum_{\substack{j_{1}= \pm 1 \\
j_{2}= \pm 1}} \chi\left(j_{1}\right) \chi\left(j_{2}\right) \\
& \times \int_{0}^{1} S_{4 m_{1}, 1+j_{1} m_{1} ; J_{m_{1}}}(\alpha) S_{4 m_{2}, 1+j_{2} m_{2} ; J_{m_{2}}}(-\alpha) d \alpha \\
& =\sum_{\substack{m_{1}, m_{2}<D \\
2\left|m_{1}, 2\right| m_{2}}} \sum_{\substack{j_{1}= \pm 1 \\
j_{2}= \pm 1}} \chi\left(j_{1}\right) \chi\left(j_{2}\right) \\
& \times \sum_{\substack{p_{i} \in J_{m_{i}, i=1,2} \\
p_{i} \equiv 1+j_{i} m_{i}\left(4 m_{i}\right), i=1,2}} \log p_{1} \log p_{2} \int_{0}^{1} e\left(\alpha\left(p_{1}-p_{2}\right)\right) d \alpha \\
& =\sum_{\substack{m<D \\
2 \mid m}} \sum_{j= \pm 1} \chi(j) \sum_{\substack{p \in J_{m} \\
p \equiv 1+j m(4 m)}}(\log p)^{2} \\
& \ll(\log N)^{2} \sum_{\substack{m<D \\
2 \mid m}} \sum_{\substack{p \in J_{m} \\
p \equiv 1+j m(4 m)}} 1 \\
& \ll N(\log N)^{2} \sum_{m<D} \frac{1}{m} \\
& \ll N \log ^{3} N \text {. }
\end{aligned}
$$


Thus from (46), (50) - (52) it follows

$$
\Gamma_{3}^{(2)}(N) \ll \frac{N^{2}}{(\log N)^{B / 2-6}} .
$$

Now let us consider $\Gamma_{3}^{(1)}(N)$. From (42) and (44) for $\nu=1$ we get

$$
\begin{aligned}
\Gamma_{3}^{(1)}(N)=\Gamma^{*} & +\mathcal{O}\left(N^{2}(\log N) \Sigma_{1}\right)+\mathcal{O}\left(\tau^{2}(\log N) \Sigma_{2}\right) \\
& +\mathcal{O}\left(N Q^{2}(\log N)^{2} \Sigma_{3}\right)+\mathcal{O}\left(N^{2} e^{-c_{0} \sqrt{\log N}} \Sigma_{4}\right)
\end{aligned}
$$

where

$$
\begin{aligned}
\Gamma^{*} & =\gamma\left(\sum_{\substack{m_{1}+m_{2}+m_{3}=N \\
m_{1} \in J_{m}}} 1\right) \sum_{\substack{m<D \\
2 \mid m}} \frac{1}{\varphi(4 m)} \sum_{j= \pm 1} \chi(j) \mathfrak{S}_{4 m, 1+j m}(N), \\
\Sigma_{1} & =\sum_{m<D} \sum_{q>Q} \frac{(4 m, q) \log q}{m q^{2}}, \\
\Sigma_{2} & =\sum_{m<D} \sum_{q \leq Q} \frac{q}{[4 m, q]}, \\
\Sigma_{3} & =\sum_{m<D} \sum_{q \leq Q} \frac{\Delta(N,[4 m, q])}{q^{2}}, \\
\Sigma_{4} & =\sum_{m<D} \frac{1}{m} .
\end{aligned}
$$

From the definition (5) it follows that $\mathfrak{S}_{4 m, 1+j m}(N)$ does not depend on $j$. Then we have $\sum_{j= \pm 1} \chi(j) \mathfrak{S}_{4 m, 1+j m}(N)=0$ and that leads to

$$
\Gamma^{*}=0 .
$$

Arguing as in [13] and using Bombieri-Vinogradov's theorem we find the following estimates

$$
\begin{aligned}
& \Sigma_{1} \ll \frac{\log ^{3} N}{Q}, \quad \Sigma_{2} \ll Q \log ^{2} N, \\
& \Sigma_{3} \ll \frac{N}{(\log N)^{A-B-5}}, \quad \Sigma_{4} \ll \log N .
\end{aligned}
$$

Bearing in mind (21), (54) - (57) we obtain

$$
\Gamma_{3}^{(1)}(N) \ll \frac{N^{2}}{(\log N)^{B-4}} .
$$

Now from (43), (53) and (58) we find

$$
\Gamma_{3}(N) \ll \frac{N^{2}}{(\log N)^{B / 2-6}} .
$$




\section{Upper bound for $\Gamma_{2}(\mathrm{~N})$}

Consider the sum $\Gamma_{2}(N)$ defined by (13). We denote by $\mathcal{F}$ the set of all primes $p \leq N$ such that $p-1$ has a divisor belongs to the interval $(D, N / D)$. Using the inequality $u v \leq u^{2}+v^{2}$ and taking into account the symmetry with respect to $d$ and $t$ we get

$$
\begin{aligned}
& \Gamma_{2}(N)^{2} \ll(\log N)^{6} N^{2-2 \gamma} \sum_{\substack{p_{1}+p_{2}+p_{3}=N \\
p_{4}+p_{5}+p_{6}=N \\
p_{2}=\left[n_{1}\right], p_{5}=\left[n_{2}\right]}}\left|\sum_{\substack{d \mid p_{1}-1 \\
D<d<N / D}} \chi(d)\right|\left|\sum_{\substack{t \mid p_{4}-1 \\
D<t<N / D}} \chi(t)\right| \\
& \ll(\log N)^{6} N^{2-2 \gamma} \sum_{\substack{p_{1}+p_{2}+p_{3}=N \\
p_{4}+p_{5}+p_{6}=N \\
p_{2}=\left[n_{c}\right], p_{5}=\left[n n_{2}\right] \\
p_{4} \in \mathcal{F}}}\left|\sum_{\substack{d \mid p_{1}-1 \\
D<d<N / D}} \chi(d)\right|^{2} .
\end{aligned}
$$

Further, we use that if $n$ is a natural such that $n \leq N$, then the number of solutions of the equation $p_{1}+p_{2}=n$ in primes $p_{1}, p_{2} \leq N$ such that $p_{1}=\left[m^{1 / \gamma}\right]$ is $\mathcal{O}\left(N^{\gamma}(\log N)^{-2} \log \log N\right)$, i.e.

$$
\#\left\{p_{1}: p_{1}+p_{2}=n, p_{1}=\left[m^{1 / \gamma}\right], \quad n \leq N\right\} \ll \frac{N^{\gamma} \log \log N}{\log ^{2} N} .
$$

This follows for example from ([3], Ch.2, Th.2.4).

Thus the summands in the sum (60) for which $p_{1}=p_{4}$ can be estimated with $\mathcal{O}\left(N^{3+\varepsilon}\right)$.

Therefore

$$
\Gamma_{2}(N)^{2} \ll(\log N)^{6} N^{2-2 \gamma} \Sigma_{1}+N^{3+\varepsilon},
$$

where

$$
\Sigma_{1}=\sum_{p_{1} \leq N}\left|\sum_{\substack{d \mid p_{1}-1 \\ D<d<N / D}} \chi(d)\right|^{2} \sum_{\substack{p_{4} \leq N \\ p_{4} \in \mathcal{F} \\ p_{4} \neq p_{1}}} \sum_{\substack{p_{2}+p_{3}=N-p_{1} \\ p_{5}+p_{6}=N-p_{4} \\ p_{2}=\left[n_{1}^{6}\right], p_{5}=\left[n_{2}\right]}} 1 .
$$

We use again (61) and find

$$
\Sigma_{1} \ll \frac{N^{2 \gamma}}{\log ^{4} N}(\log \log N)^{2} \Sigma_{2} \Sigma_{3},
$$

where

$$
\Sigma_{2}=\sum_{p \leq N}\left|\sum_{\substack{d \mid p-1 \\ D<d<N / D}} \chi(d)\right|^{2}, \quad \Sigma_{3}=\sum_{\substack{p \leq N \\ p \in \mathcal{F}}} 1 .
$$

Arguing as in ([4], Ch.5) we find

$$
\Sigma_{2} \ll \frac{N(\log \log N)^{7}}{\log N}, \quad \Sigma_{3} \ll \frac{N(\log \log N)^{3}}{(\log N)^{1+2 \theta_{0}}} .
$$

where $\theta_{0}$ is denoted by (4).

From (62) - (64) it follows

$$
\Gamma_{2}(N) \ll N^{2}(\log N)^{-\theta_{0}}(\log \log N)^{6}
$$




\section{Asymptotic formula for $\Gamma_{1}(\mathrm{~N})$}

In this section our argument is a modification of Tolev's [14] argument.

Consider the sum $\Gamma_{1}(N)$. From (12), (15) and (22) we get

$$
\Gamma_{1}(N)=\Gamma_{1}^{(1)}(N)+\Gamma_{1}^{(2)}(N),
$$

where

$$
\begin{aligned}
& \Gamma_{1}^{(1)}(N)=\sum_{d \leq D} \chi(d) I_{d, 1}^{(1)}(N), \\
& \Gamma_{1}^{(2)}(N)=\sum_{d \leq D} \chi(d) I_{d, 1}^{(2)}(N) .
\end{aligned}
$$

We estimate the sum $\Gamma_{1}^{(2)}(N)$ by the same way as the sum $\Gamma_{3}^{(2)}(N)$ and obtain

$$
\Gamma_{1}^{(2)}(N) \ll \frac{N^{2}}{(\log N)^{B / 2-6}} .
$$

Now we consider $\Gamma_{1}^{(1)}(N)$. We use the formula (42) for $J=[1, N]$. The error term is estimated by the same way as for $\Gamma_{3}^{(1)}(N)$. We have

$$
\Gamma_{1}^{(1)}(N)=\frac{\gamma}{2} \mathfrak{S}(N) N^{2} \sum_{d \leq D} \frac{\chi(d) \mathfrak{S}_{d, 1}^{*}(N)}{\varphi(d)}+\mathcal{O}\left(\frac{N^{2}}{(\log X)^{B-4}}\right),
$$

where $\mathfrak{S}(N)$ is defined by (6) and

$$
\begin{aligned}
\mathfrak{S}_{d, 1}^{*}(N)= & \prod_{\substack{p|d \\
p| N}}\left(1-\frac{1}{(p-1)^{2}}\right)^{-1} \prod_{\substack{p \mid d \\
p \nmid N-1}}\left(1-\frac{1}{(p-1)^{2}}\right) \\
& \times \prod_{\substack{p \mid d \\
p \nmid N}}\left(1+\frac{1}{(p-1)^{3}}\right)^{-1} \prod_{\substack{p|d \\
p| N-1}}\left(1+\frac{1}{p-1}\right) ;
\end{aligned}
$$

Denote

$$
\Sigma=\sum_{d \leq D} f(d), \quad f(d)=\frac{\chi(d) \mathfrak{S}_{d, 1}^{*}(N)}{\varphi(d)}
$$

We have

$$
f(d) \ll d^{-1}(\log \log (10 d))^{2}
$$

with absolute constant in the Vinogradov's symbol. Hence the corresponding Dirichlet series

$$
F(s)=\sum_{d=1}^{\infty} \frac{f(d)}{d^{s}}
$$

is absolutely convergent in $\operatorname{Re}(s)>0$. On the other hand, $f(d)$ is multiplicative with respect to $d$ and applying Euler's identity we find

$$
F(s)=\prod_{p} T(p, s), \quad T(p, s)=1+\sum_{l=1}^{\infty} f\left(p^{l}\right) p^{-l s} .
$$


From (69), (70) and (72) we establish that

$$
T(p, s)=\left(1-\frac{\chi(p)}{p^{s+1}}\right)^{-1}\left(1+\frac{\chi(p)}{p^{s+1}} E_{d}(p)\right),
$$

where

$$
E_{d}(p)= \begin{cases}(p-3)\left(p^{2}-3 p+3\right)^{-1} & \text { if } p \nmid N(N-1), \\ (p-1)^{-1} & \text { if } p \mid N \\ (2 p-3)\left(p^{2}-3 p+3\right)^{-1} & \text { if } p \mid N-1 .\end{cases}
$$

Hence we find

$$
F(s)=L(s+1, \chi) \mathcal{N}(s),
$$

where $L(s+1, \chi)$ is Dirichlet series corresponding to the character $\chi$ and

$$
\begin{aligned}
\mathcal{N}(s)= & \prod_{p \nmid N(N-1)}\left(1+\chi(p) \frac{p-3}{p^{s+1}\left(p^{2}-3 p+3\right)}\right) \prod_{p \mid N}\left(1+\chi(p) \frac{1}{p^{s+1}(p-1)}\right) \\
& \times \prod_{p \mid N-1}\left(1+\chi(p) \frac{2 p-3}{p^{s+1}\left(p^{2}-3 p+3\right)}\right) .
\end{aligned}
$$

From the properties of the $L$-functions it follows that $F(s)$ has an analytic continuation to $\operatorname{Re}(s)>-1$. It is well known that

$$
L(s+1, \chi) \ll 1+|\operatorname{Im}(s)|^{1 / 6} \quad \text { for } \operatorname{Re}(s) \geq-\frac{1}{2} .
$$

Moreover,

$$
\mathcal{N}(s) \ll 1
$$

Using (73), (75) and (76) we get

$$
F(s) \ll N^{1 / 6} \text { for } \operatorname{Re}(s) \geq-\frac{1}{2}, \quad|\operatorname{Im}(s)| \leq N .
$$

We apply Perron's formula given at Tenenbaum ([11], Chapter II.2) and also (71) to obtain

$$
\Sigma=\frac{1}{2 \pi \imath} \int_{\varkappa-\imath N}^{\varkappa+\imath N} F(s) \frac{D^{s}}{s} d s+\mathcal{O}\left(\sum_{t=1}^{\infty} \frac{D^{\varkappa} \log \log (10 t)}{t^{1+\varkappa}\left(1+N\left|\log \frac{D}{t}\right|\right)}\right)
$$

where $\varkappa=1 / 10$. It is easy to see that the error term above is $\mathcal{O}\left(N^{-1 / 20}\right)$.

Applying the residue theorem we see that the main term in (78) is equal to

$$
F(0)+\frac{1}{2 \pi \imath}\left(\int_{1 / 10-\imath N}^{-1 / 2-\imath N}+\int_{-1 / 2-\imath N}^{-1 / 2+\imath N}+\int_{-1 / 2+\imath N}^{1 / 10+\imath N}\right) F(s) \frac{D^{s}}{s} d s .
$$

From (77) it follows that the contribution from the above integrals is $\mathcal{O}\left(N^{-1 / 20}\right)$.

Hence

$$
\Sigma=F(0)+\mathcal{O}\left(N^{-1 / 20}\right)
$$


Using (73) we get

$$
F(0)=\frac{\pi}{4} \mathcal{N}(0)
$$

Bearing in mind (68), (70), (74), (79) and (80) we find a new expression for $\Gamma_{1}^{(1)}(N)$

$$
\Gamma_{1}^{(1)}(N)=\frac{\gamma}{8} \mathfrak{S}_{\Gamma}(N) N^{2}+\mathcal{O}\left(\frac{N^{2}}{(\log N)^{B-4}}\right),
$$

where $\mathfrak{S}_{\Gamma}$ is defined by (7).

From (66), (67) and (81) we obtain

$$
\Gamma_{1}(N)=\frac{\gamma}{8} \mathfrak{S}_{\Gamma}(N) N^{2}+\mathcal{O}\left(\frac{N^{2}}{(\log N)^{B / 2-6}}\right) .
$$

\section{Proof of the Theorem}

Therefore using (11), (59), (65) and (82) we find

$$
\Gamma(N)=\frac{\gamma}{2} \mathfrak{S}_{\Gamma}(N) N^{2}+\mathcal{O}\left(N^{2}(\log N)^{-\theta_{0}}(\log \log N)^{6}\right)
$$

This implies that $\Gamma(N) \rightarrow \infty$ as $N \rightarrow \infty$.

The Theorem is proved.

\section{References}

[1] Balog, A. \& Friedlander, J. P.(1992) A hybrid of theorems of Vinogradov and PiatetskiShapiro, Pacific J. Math., 156, 45-62.

[2] Dimitrov, S. I. (2017) Prime triples $p_{1}, p_{2}, p_{3}$ in arithmetic progressions such that $p_{1}=$ $x^{2}+y^{2}+1, p_{3}=\left[n^{c}\right]$, Notes on Number Theory and Discrete Mathematics, 23(4), 22-33.

[3] Halberstam, H. \& Richert , H.-E. (1974) Sieve Methods, Academic Press.

[4] Hooley, C. (1976) Applications of sieve methods to the theory of numbers, Cambridge Univ. Press.

[5] Jia, C.-H. (1995) On the Piatetski-Shapiro-Vinogradov theorem, Acta Arith., 73, 1-28.

[6] Karatsuba, A. (1983) Principles of the Analytic Number Theory, Nauka, Moscow, (in Russian).

[7] Kumchev, A. (1997) On the Piatetski-Shapiro-Vinogradov Theorem, Journal de Théorie des Nombres de Bordeaux, 9, 11-23.

[8] Linnik, Ju. (1960) An asymptotic formula in an additive problem of Hardy and Littlewood, Izv. Akad. Nauk SSSR, Ser.Mat., 24, 629-706 (in Russian). 
[9] Piatetski-Shapiro, I. I. (1953) On the distribution of prime numbers in sequences of the form $[f(n)]$, Mat. Sb., 33, 559-566.

[10] Rivat, J. \& Wu, J. (2001) Prime numbers of the form [n $n^{c}$, Glasg. Math. J, 43(2), 237-254.

[11] Tenenbaum, G. (1995) Introduction to Analytic and Probabilistic Number Theory, Cambridge Univ. Press.

[12] Teräväinen, J. (2018) The Goldbach problem for primes that are sums of two squares plus one, Mathematika, 64(1), 20-70.

[13] Tolev, D. (1997) On the number of representations of an odd integer as a sum of three primes, one of which belongs to an arithmetic progression, Proc. Steklov Math. Inst., 218, 415-432.

[14] Tolev, D. (2010) The ternary Goldbach problem with arithmetic weights attached to one of the variables, J. Number Theory, 130, 439-457.

[15] Vinogradov, I. M. (1937) Representation of an odd number as the sum of three primes, Dokl. Akad. Nauk. SSSR, 15, 291-294 (in Russian). 